Courrier du Centre international Blaise Pascal

$11 \mid 1989$

Varia

\title{
Les expériences du vide dans le vide
}

\section{Kimiyo Koyanagi}

\section{OpenEdition}

\section{Journals}

Édition électronique

URL : http://journals.openedition.org/ccibp/642

DOI : $10.4000 /$ ccibp. 642

ISSN : 2493-7460

\section{Éditeur}

Centre international Blaise Pascal

\section{Édition imprimée}

Date de publication : 20 décembre 1989

Pagination : $3-23$

ISSN : 0249-6674

\section{Référence électronique}

Kimiyo Koyanagi, «Les expériences du vide dans le vide», Courrier du Centre international Blaise Pascal [En ligne], 11 | 1989, mis en ligne le 10 décembre 2015, consulté le 19 avril 2019. URL : http:// journals.openedition.org/ccibp/642 ; DOI : 10.4000/ccibp.642

Ce document a été généré automatiquement le 19 avril 2019.

Centre international Blaise Pascal 


\section{Les expériences du vide dans le vide}

Kimiyo Koyanagi

\section{I.-.L'expérience du vide dans le vide d'après le Traité de la pesanteur de la masse de l'air (Chapitre VI) reconstituée par Akira Kurobé et Kimiyo Koyanagi au laboratoire national de métrologie (Tokyo)}

1 Ce dispositif est le plus simple de tous. L'édition originale du traité de Pascal présente une figure défectueuse (Euvres complètes, édition Jean Mesnard, II, p. 1087, et le commentaire p. 1042). Malgré son élégance et la simplicité de son principe, nous pensons que ce dispositif correspond à une expérience de pensée : sa réalisation n'est confirmée par aucun témoignage ; et même après rectification de l'erreur de la figure originale, il n'est pas possible de la réaliser avec le dispositif que présente Pascal (voir K. Koyanagi, Pascal et l'expérience du vide dans le vide). 
Plan dessiné par M. Kurobé pour le dispositif du vide dans le vide de La Pesanteur de la Masse de l'Air, restitué en 1974 par M. Akira Kurobé au Laboratoire National de Métrologie de Tokyo.

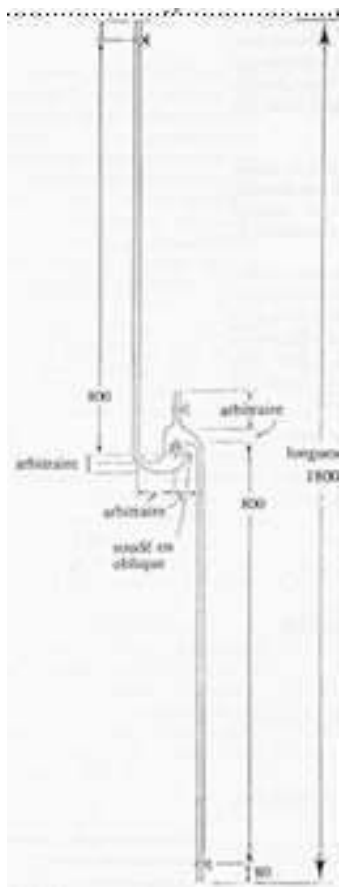

Diamètre du tuyau :

Tuyau étroit, diamètre extérieur : $10 \mathrm{~mm}$; diamètre intérieur : $6 \mathrm{~mm}$.

Tuyau large, diamètre extérieur : $40 \mathrm{~mm}$; diamètre intérieur : 35,2 mm.

\section{Le dispositif de Pascal}

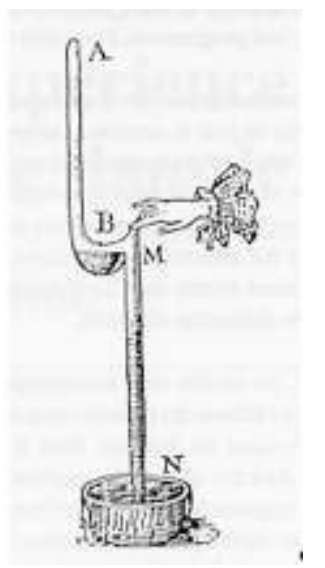

Cuvres complètes de Pascal, éd. Jean Mesnard, II, p. 1087 :

«Il faut avoir un tuyau recourbé par en bas, bouché par le bout A, et ouvert par le bout $\mathrm{B}$, et un autre tuyau tout droit, ouvert par les deux bouts, $\mathrm{M}$ et $\mathrm{N}$, mais inséré et soudé par le bout $\mathrm{M}$ dans le bout recourbé de l'autre, comme il paraît en cette figure (...). » 


\section{Dispositif de vif-argent}

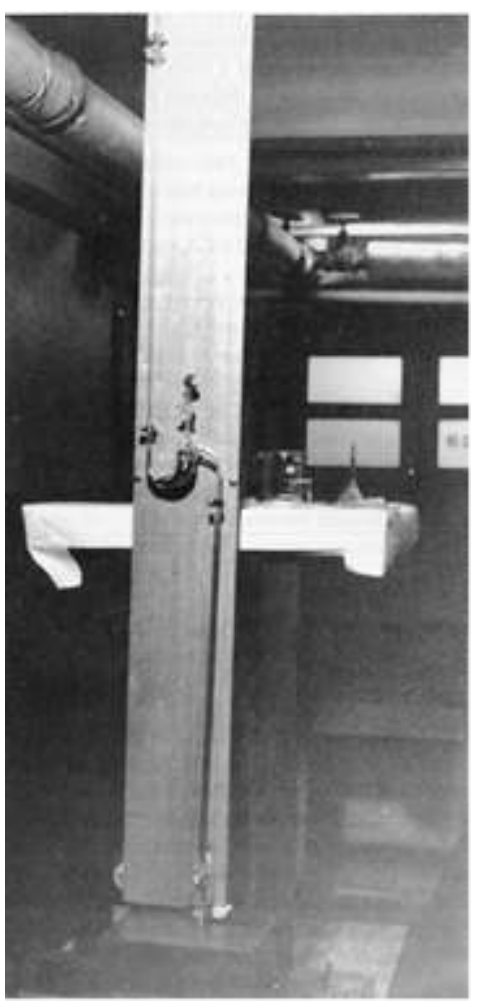

Étape préparatoire : M. Kurobé remplit le dispositif de vif argent

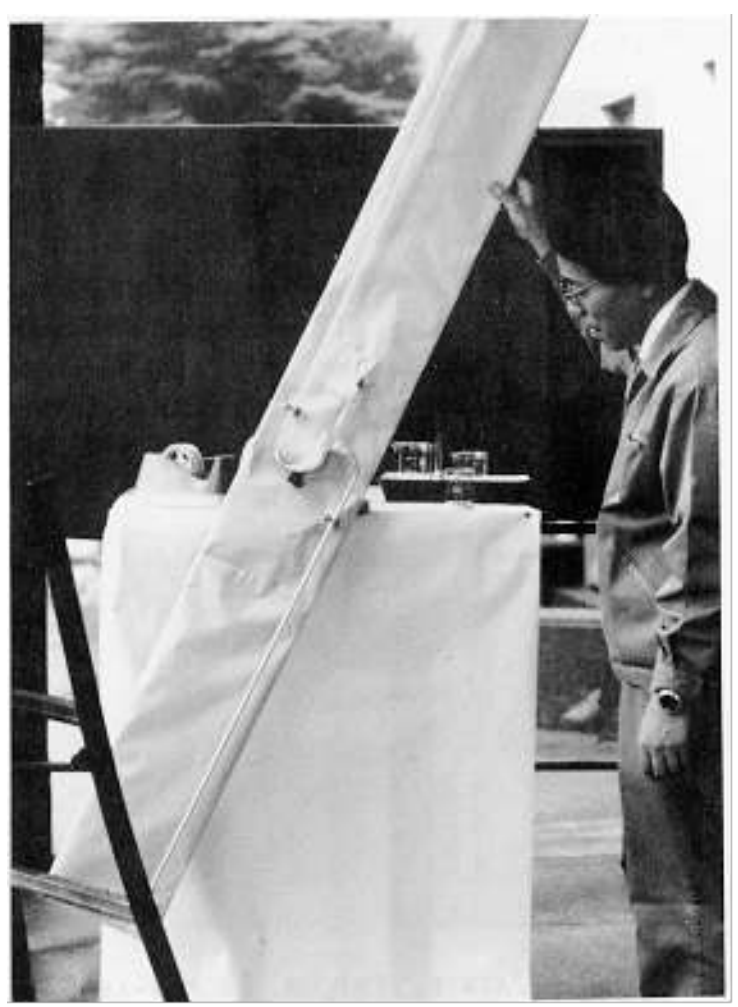




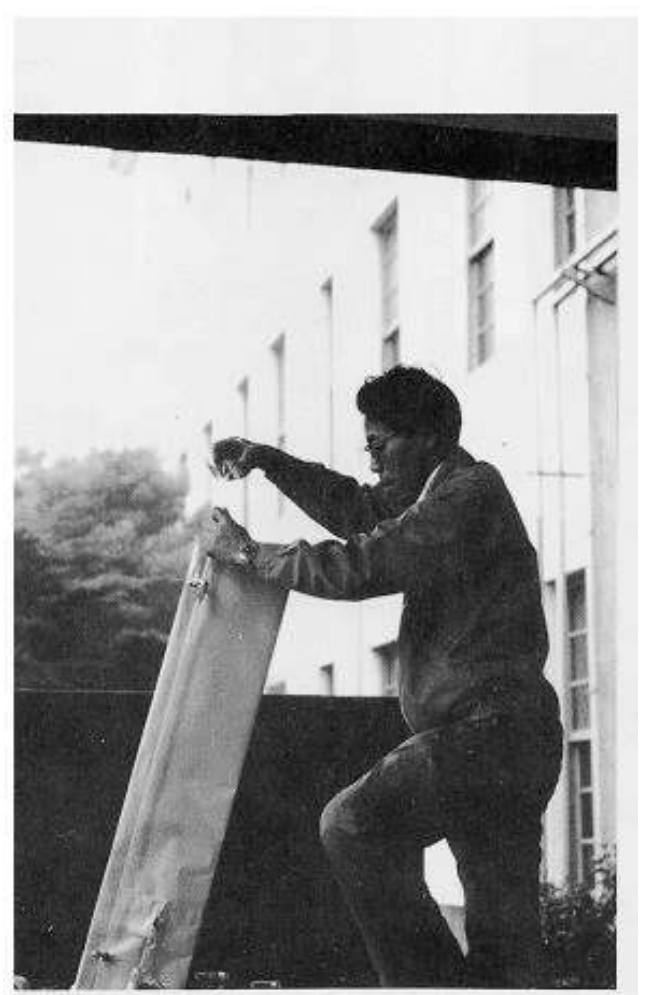

$3 \quad$ Ibid., p. 1087 :

«Il faut boucher B, qui est l'ouverture du bout recourbé du premier tuyau, avec le doigt ou autrement, comme avec une vessie de pourceau, et renverser ce tuyau entier ; c'est-à-dire les deux tuyaux qui n'en font proprement qu'un, puisqu'ils ont communication l'un dans l'autre ; le remplir de vif-argent (...).»

\section{Le dispositif rempli de vif-argent}

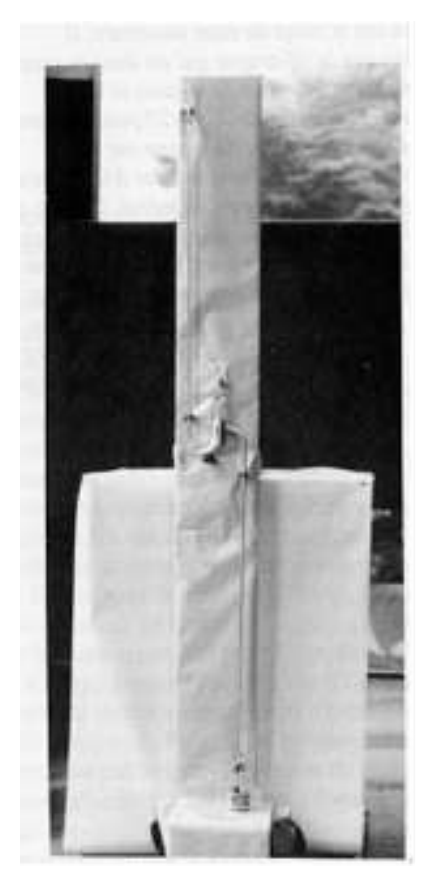


Première étape. Le robinet du tuyau inférieur est ouvert

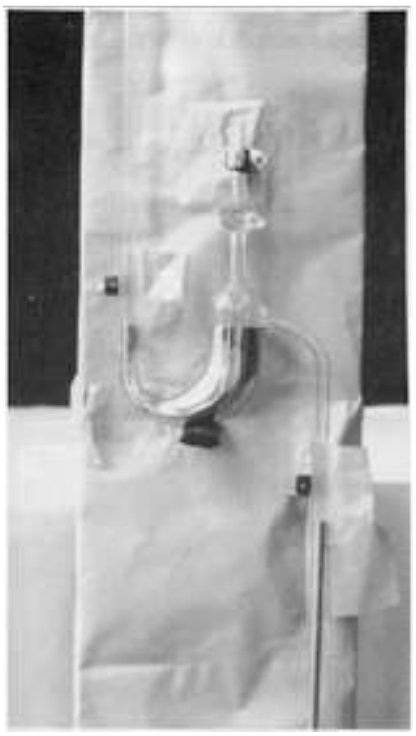

Ibid., p. 1087-1088 :

Il arrivera que le vif argent du tuyau d'en haut tombera entièrement, et sera tout reçu dans sa recourbure, si ce n'est qu'il y en aura une partie qui s'écoulera dans le tuyau d'en bas par le trou M ; mais le vif-argent du tuyau d'en bas tombera en partie seulement, et demeurera suspendu aussi en partie, à hauteur d'environ 26 à 27 pouces, suivant le lieu et le temps où l'on en fait l'épreuve. Or la raison de cette différence est que l'air pèse sur le vif-argent qui est dans l'écuelle au bout du tuyau d'en bas; et ainsi il tient son vif-argent du dedans suspendu et en équilibre ; mais il ne pèse pas sur le vif-argent qui est au bout recourbé du tuyau d'en haut; car le doigt ou la vessie qui le bouche empêchent qu'il n'y ait d'accès; de sorte que, comme il n'y a aucun air qui pèse en cet endroit, le vif argent du tuyau tombe librement, parce que rien ne le soutient et ne s'oppose à sa chute [...]. 
Deuxième étape. K. Koyanagi tourne le robinet et fait entrer l'air extérieur. Le vif-argent monte dans le tuyau.

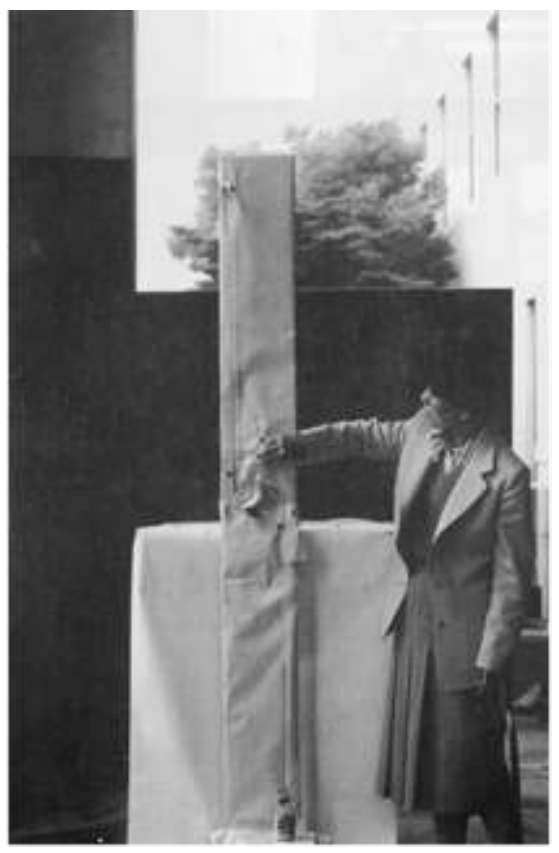

Ibid., p. 1088 :

Et si on ôte le doigt de cette ouverture, il arrivera que le vif-argent qui est dans la recourbure montera tout d'un coup dans le tuyau jusques à la hauteur de 26 ou 27 pouces, parce que l'air, tombant tout d'un coup sur le vif argent, le fera incontinent monter à la hauteur capable de le contrepeser, et même, à cause de la violence de sa chute, il le fait monter un peu au-delà de ce terme ; mais il tombera ensuite un peu plus bas, et puis il remontera encore; et après quelques allées et venues, comme d'un poids suspendu au bout d'un fil, il demeurera ferme à une certaine hauteur, à laquelle il contrepèse l'air précisément.

\section{Les quatre dispositifs de l'expérience du vide dans le vide : l'expérience du vide dans le vide selon Pascal, Roberval, Auzoult et Rohault.}

Commentaire : Ces quatre expériences ont été reconstituées en 1982 par Kimiyo Koyanagi et Masao Uchida, d'après les descriptions originales, au Laboratoire de transformation du verre de M. Shigeru Ando (Tokyo). Seul l'emploi de la vessie de porc pour boucher les extrémités des tuyaux s'est avéré impossible, à cause de la grande pression exercée par le mercure. Il a fallu remplacer les vessies par des bouchons de caoutchouc utilisés pour la chromatographie des gaz. De ces modifications inévitables, les rapports originaux ne font presque jamais mention; il ne faudrait cependant pas en conclure qu'il s'agit d'expériences de pensée, car il faut tenir compte du style des descriptions d'expériences au XVII siècle et du fait que les rapporteurs ne fournissent pas toujours les détails. (D'après le rapport présenté par les auteurs à l'occasion de la vingt-neuvième assemblée de la Société Japonaise d'Histoire des Sciences). 
Les quatre dispositifs

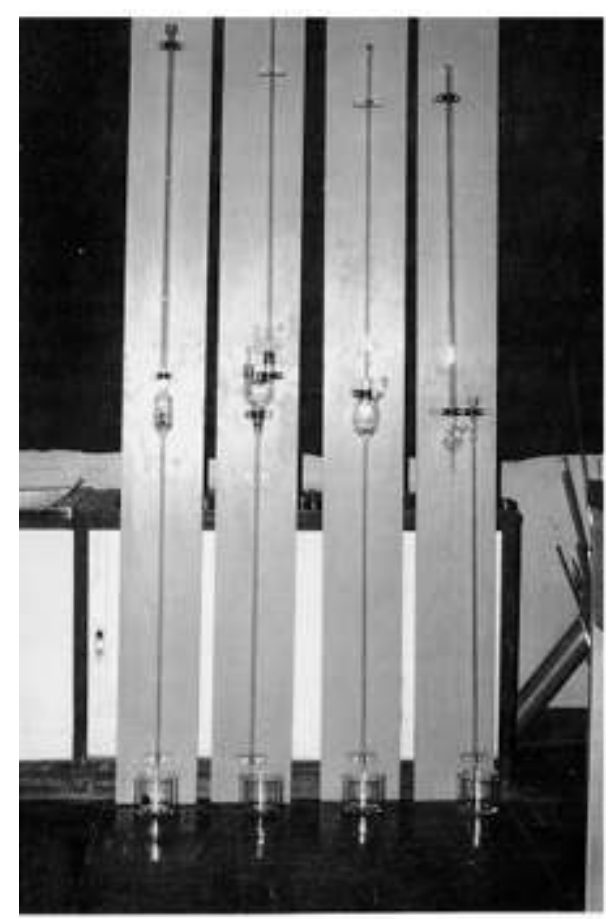

\section{L'expérience du vide dans le vide d'après Pascal}

7 Commentaire: Pascal fait allusion à cette expérience dans sa lettre à Périer du 15 novembre 1647, Euvres complètes de Pascal, éd. J. Mesnard, II, p. 679. Le P. Noël donne dans la Gravitas comparata une description plus précise (voir Euvres complètes, II, p. 635-636). !"

Ce dispositif est le plus difficile à réaliser. La plus grande difficulté consiste à « déboucher l'orifice intérieur » du petit tuyau : cet orifice doit être à la fois fermé par une peau de vessie très serrée et facile à dénouer du dehors. Le vif-argent empêche évidemment de voir l'extrémité du petit tube, et le grand tube interdit de la manipuler.

9 Après avoir fini le remplissage du grand tube, l'appareil apparaît comme un bâton d'argent, dont on ne peut pas apercevoir l'intérieur. Au fur et à mesure qu'on remplit le grand tube de vif-argent, le petit tube intérieur flotte dans le grand tube, car le poids spécifique du verre est inférieur à celui du vif-argent. Il a fallu le repousser sans cesse dans le grand tube. 
Extrémité inférieure du petit tuyau

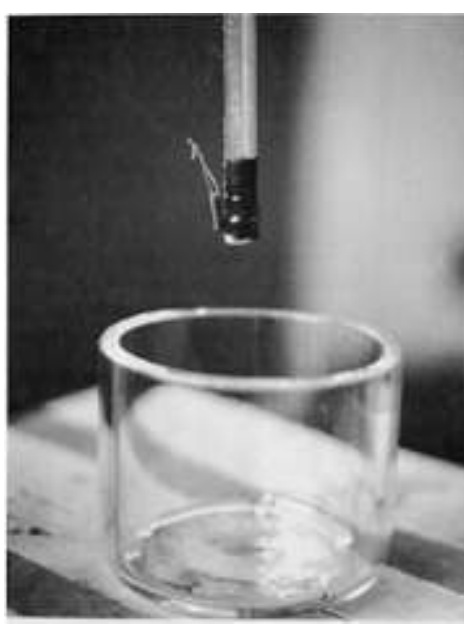

Ibid., p. 635 :

[...] Soit un tube de verre de trois pieds, plein de vif-argent, et fermé à chaque extrémité par une peau de vessie ; [...]

11 Commentaire : Nous avons d'abord essayé de fermer les deux extrémités du tuyau par une pellicule au lieu d'une peau de vessie (figure 1).

Figure 1

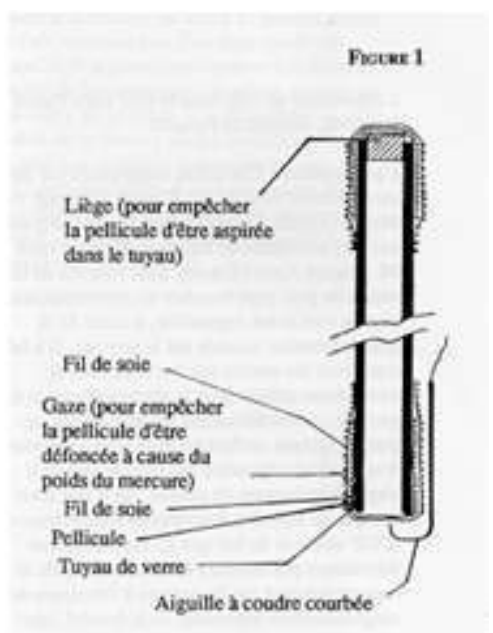

12 Le bout inférieur a été équipé d'une aiguille à coudre courbée comme un hameçon, de sorte que l'aiguille, perçant la pellicule, fasse sortir le mercure du tuyau dans son vase (figure 2). 
Figure 2

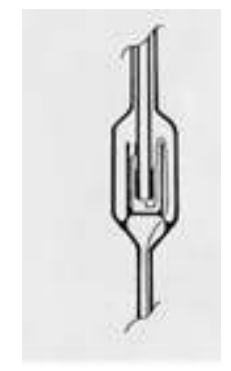

13 Mais il était très difficile de fermer complètement l'orifice inférieur du tuyau : le mercure en sort inévitablement. On a donc renoncé à l'emploi de la pellicule et employé des capsules en gomme silicone.

14 L'orifice inférieur montré sur la photo est équipé d'une aiguille de seringue courbée, de sorte que le petit fourreau de gomme, qui couvre l'extrémité la plus large de l'aiguille, soit ôté quand on tire le fil (figure 3).

Figure 3

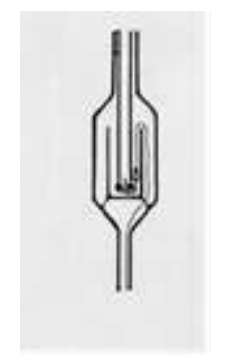

Installation du petit tuyau dans le long tuyau

Ibid., p. 635 :

[...] soit ce tube avec son vase introduit dans un autre tube de six pieds, fermé à son extrémité inférieure par une membrane, en sorte que le vase soit fixé à la partie supérieure du long tube ; [...]

Commentaire : On voit le fil rouge, qui, fixé par l'aiguille, sort par l'extrémité inférieure du long tuyau.

Le fil rouge, fixé par l'aiguille sort par l'extrémité inférieure du long tuyau

Remplissage du vase intérieur par l'extrémité supérieure du long tuyau

Ibid., p. 635 :

[...] soit l'extrémité bouchée du grand tube élevé perpendiculairement plongée dans un récipient plein de vif-argent ; soit le tube lui-même rempli de vif-argent ; [...]

Remplissage du long tuyau par son extrémité supérieure

Appareil complètement rempli de mercure : étape préparatoire

Ibid., p. 635 : [...] soit fermée son extrémité supérieure [...] 
Étape préparatoire : appareil complètement rempli de mercure

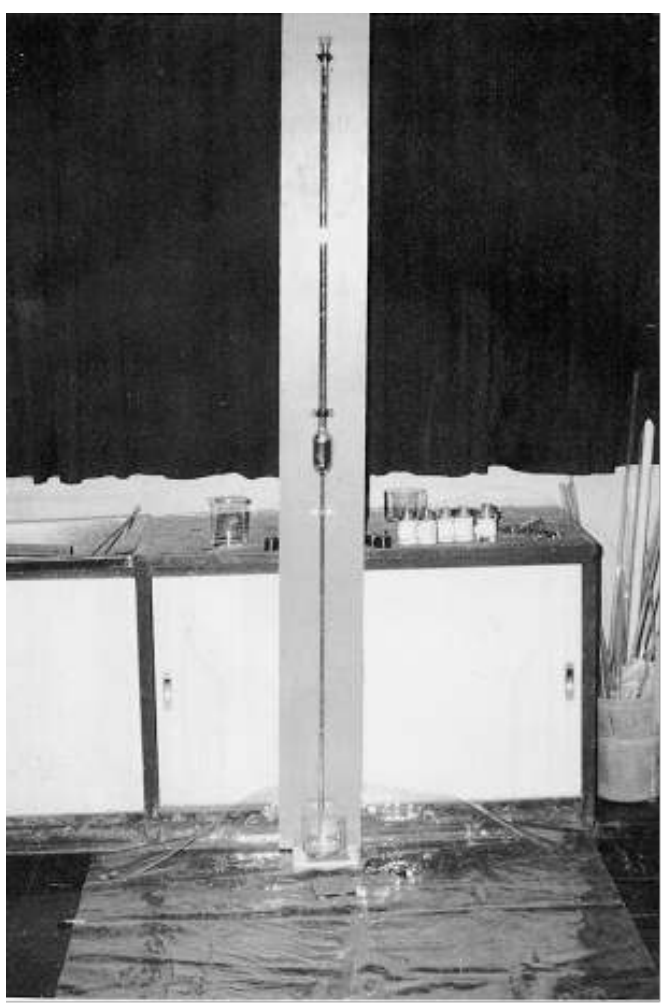

Ouverture du gros tuyau par l'extrémité inférieure

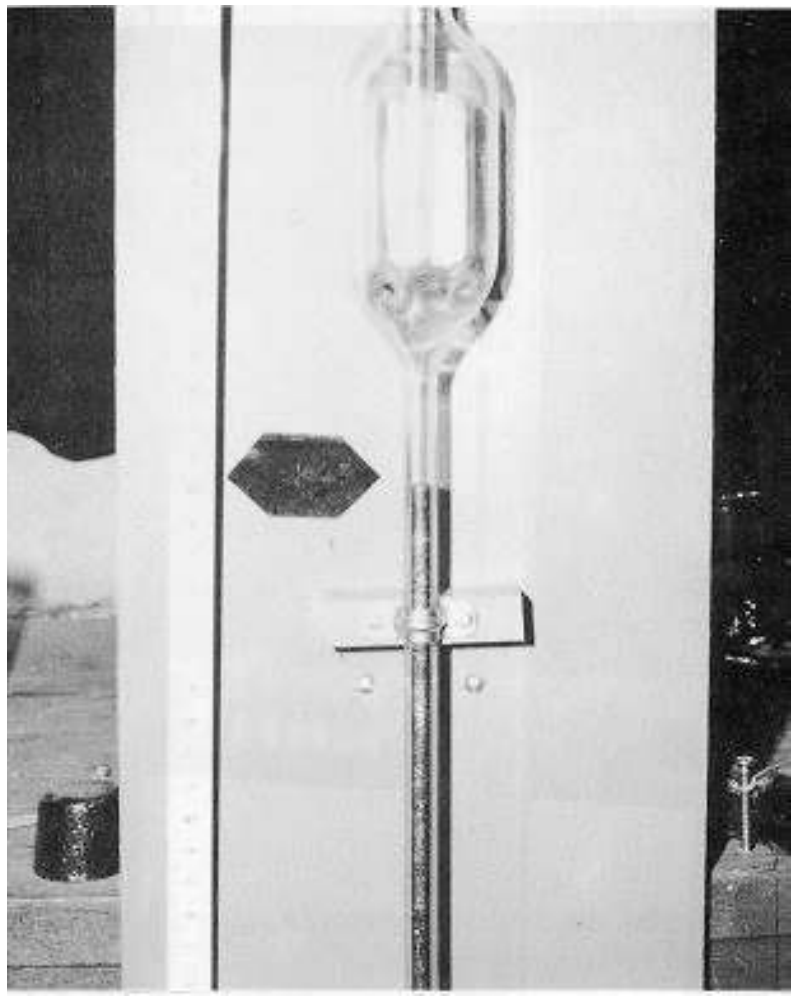

18 Ibid., p. 635: 
[...] et ouverte l'inférieure ; le vif-argent descendra jusqu'à la hauteur de vingt-sept pouces. Voilà [...] le petit tube plein de vif-argent : [...]

\section{Appareil complètement rempli de mercure : étape préparatoire}

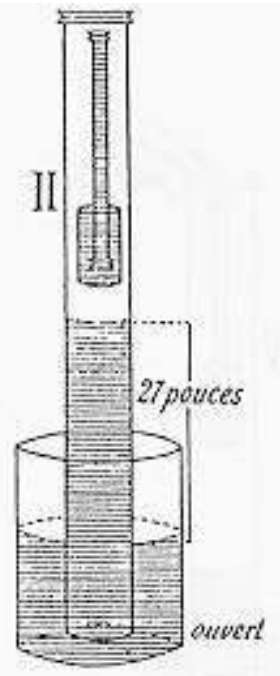

Ibid., p. 635 :

[...] soit fermée son extrémité supérieure,[...]

Ouverture du gros tuyau par l'extrémité inférieure

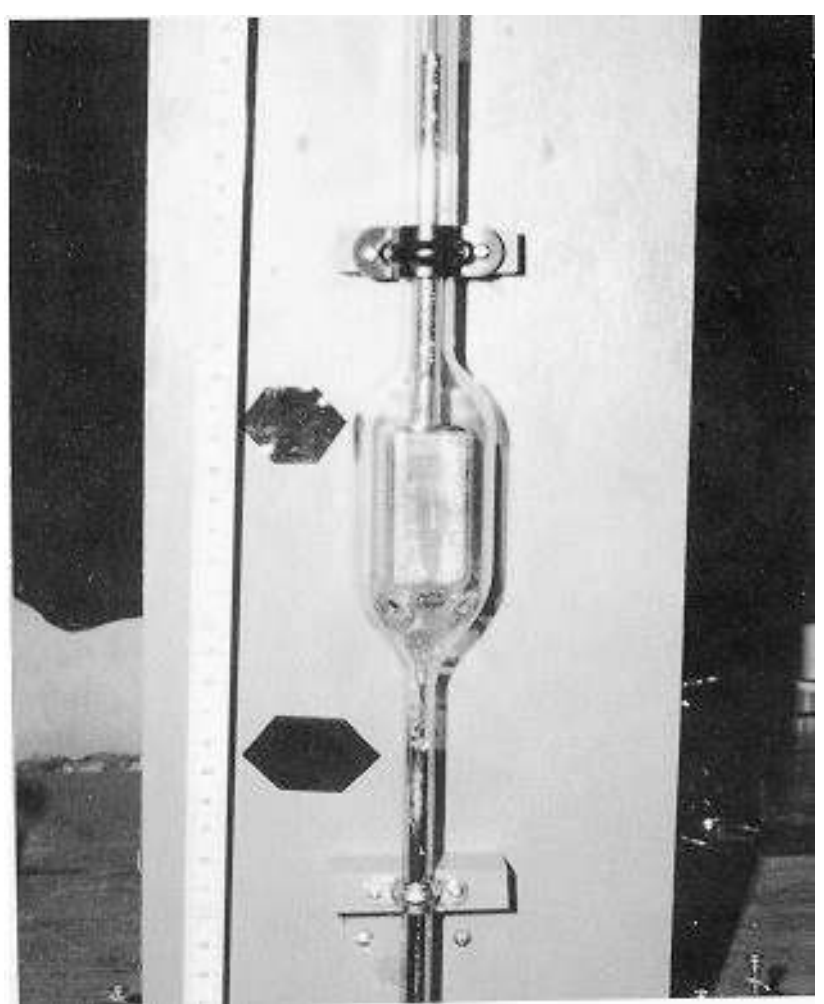

Ibid., p. 635 :

[...] et ouverte l'inférieure ; le vif-argent descendra jusqu'à la hauteur de vingt-sept pouces. Voilà [...] le petit tube plein de vif-argent : [...]

La pointe indique le niveau de mercure dans le tube. 
Ouverture du petit tuyau par l'extrémité inférieure

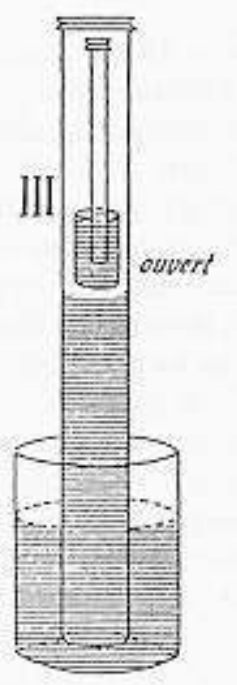

Ibid., p. 635-636

[...] si l'on débouche son orifice inférieur maintenu plongé dans le vase, tout le vifargent coulera en bas

Commentaire : On tire le fil de sorte que l'aiguille perce la capsule.

Introduction d'air extérieur par le petit trou d'une aiguille de seringue : deuxième étape

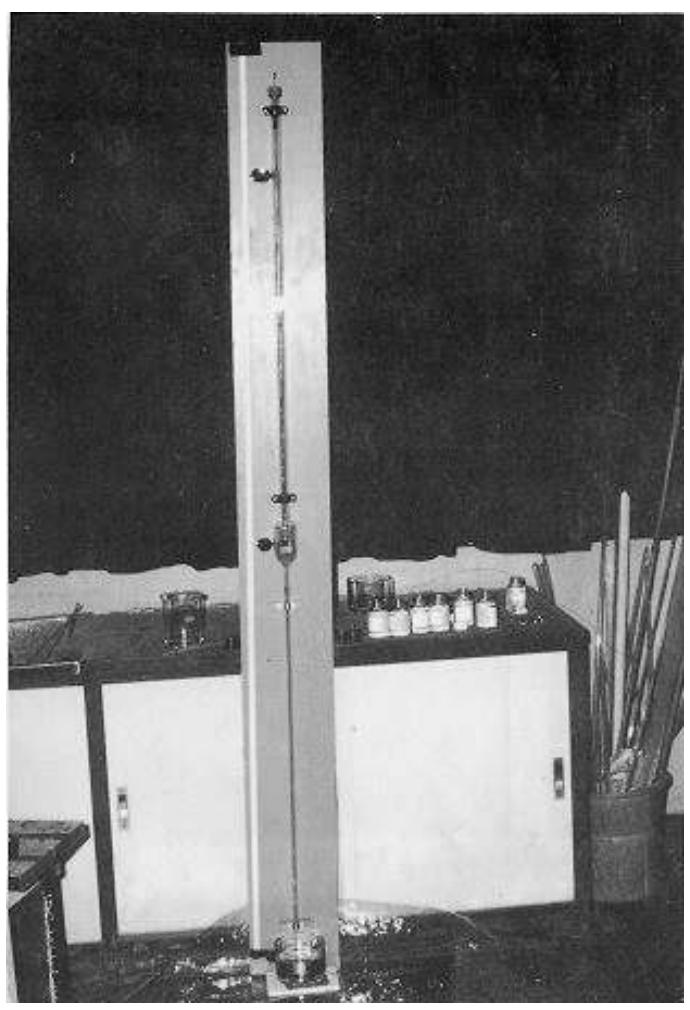




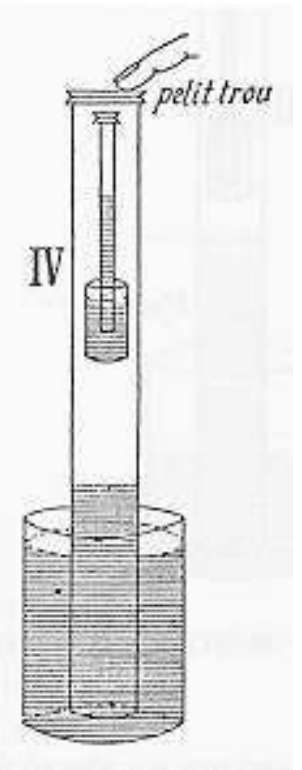

21 Ibid., p. 636

Par un trou étroit, que l'on fermera aussitôt avec le doigt, qu'on laisse un peu d'air s'écouler dans le grand tube : alors le vif-argent qui est dans le vase du petit tube refluera dans son tube ; et celui qui est dans le long tube descend.

\section{L'expérience d'Auzoult}

L'expérience d'Auzoult a été décrite dans la Dissertatio anatomica de Circulatione Sanguinis et Chyli Motu de Pecquet (1651). Voir ce passage dans Euvres complètes de Pascal, éd. J. Mesnard, p. 768-771

Montage de l'appareil

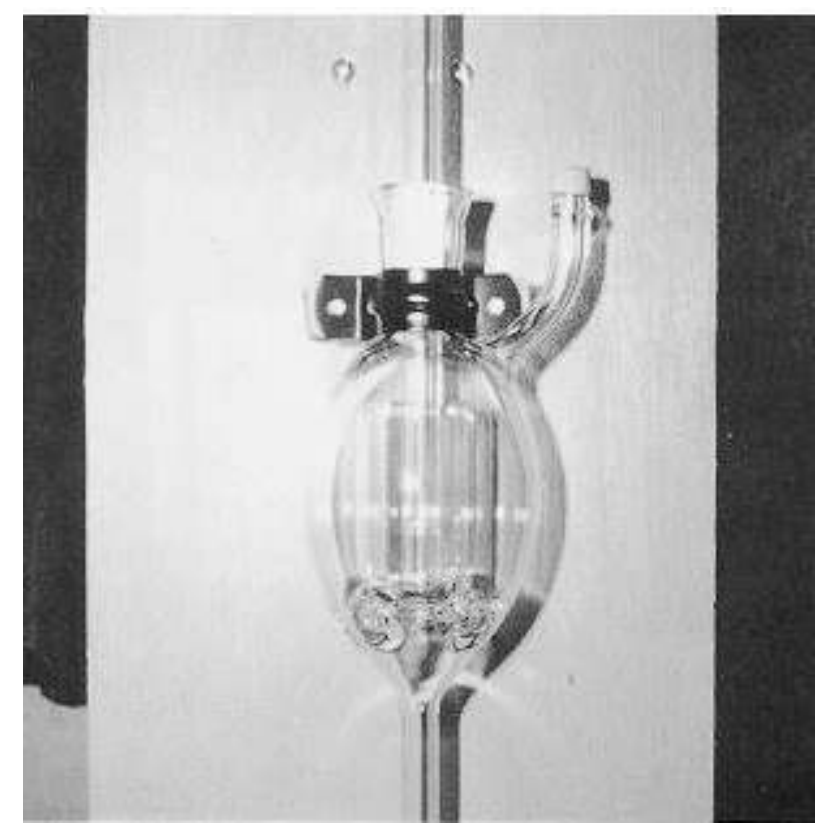



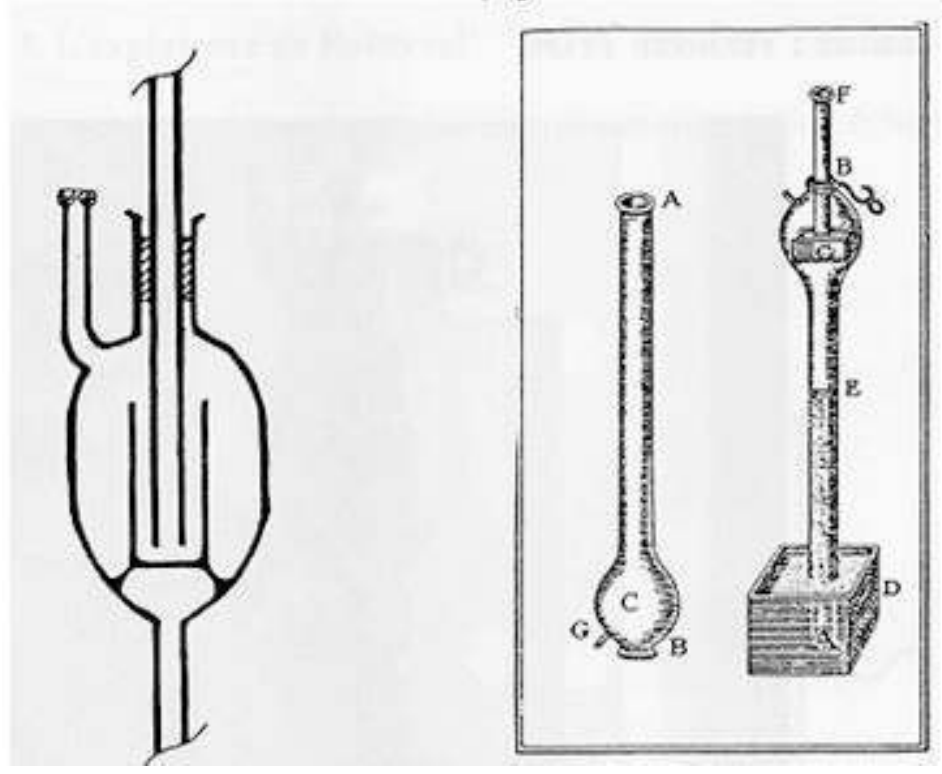

Ibid., p. 768 :

Prends un flacon $\mathrm{AB}[\ldots]$ prolongé par un col [...] si ce n'est qu'est fixé près de la base $B$ un petit conduit supplémentaire $G$, par l'orifice duquel, chaque fois que besoin sera, un accès puisse s'ouvrir à l'air dans le flacon. Retourne la base $B$ et place-la en haut, puis, par son embouchure ouverte, introduis un vase carré $C$ d'assemblage en parallélépipède de manière que le réceptacle, sa partie creuse tournée vers le sommet ouvert de la base B, repose horizontalement par son fond sur le tuyau de dessous formé par le col AC, qui lui est perpendiculaire. Bien entendu, le vase $C$, appuyé intérieurement sur le verre par ses quatre angles externes, laissera une voie de descente sous forme d'arcs; autrement dit, entre les côtés du vase et le flacon il restera un intervalle ouvert. Enfonce dans le vase $C$, vertical ou bien droit, un tube $\mathrm{CF}$, de longueur voulue, également en verre, ayant ouverture aux deux bouts à la fois; entoure-le d'une vessie de porc très soigneusement nouée (avec laquelle tu boucheras également la base et l'orifice $G$ ), pour éliminer l'air en $\mathrm{B} ;[. .$. 
Appareil complètement rempli de mercure : étape préparatoire

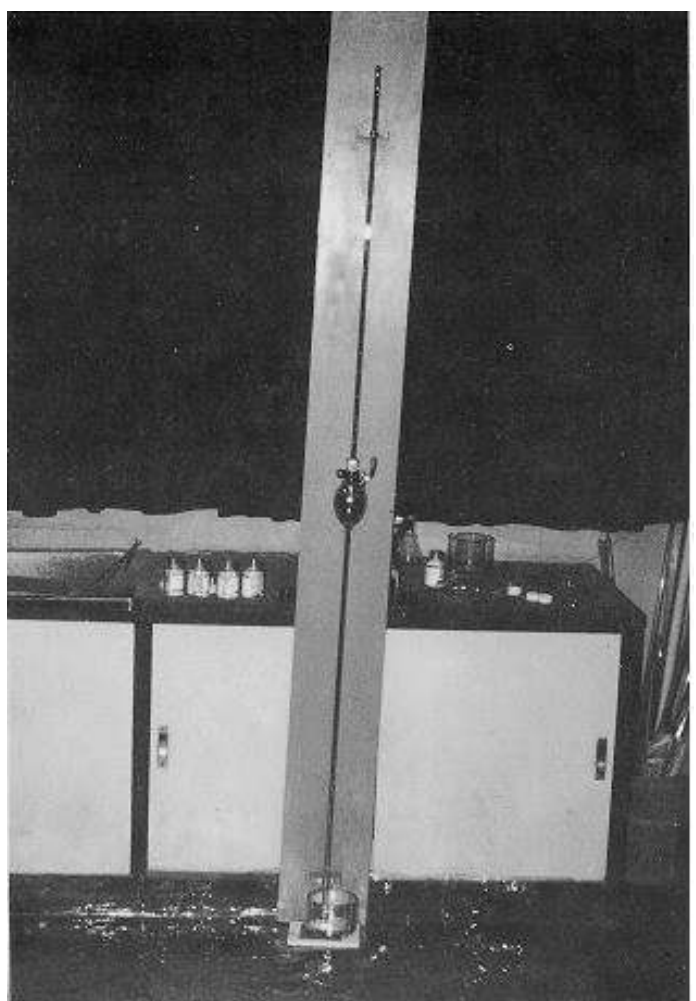

Ibid., p. 769 :

[...] tu prieras alors l'aide qui te sert de placer son doigt sur l'ouverture A du col du flacon et de le tenir bouché pendant tout le temps que tu verseras du vif-argent et en rempliras tout l'instrument par le sommet ouvert du tube complémentaire $\mathrm{F}$; clos en fin aussi ce sommet par une membrane de porc : [...]

Ouverture du tube par l'extrémité inférieure : première étape

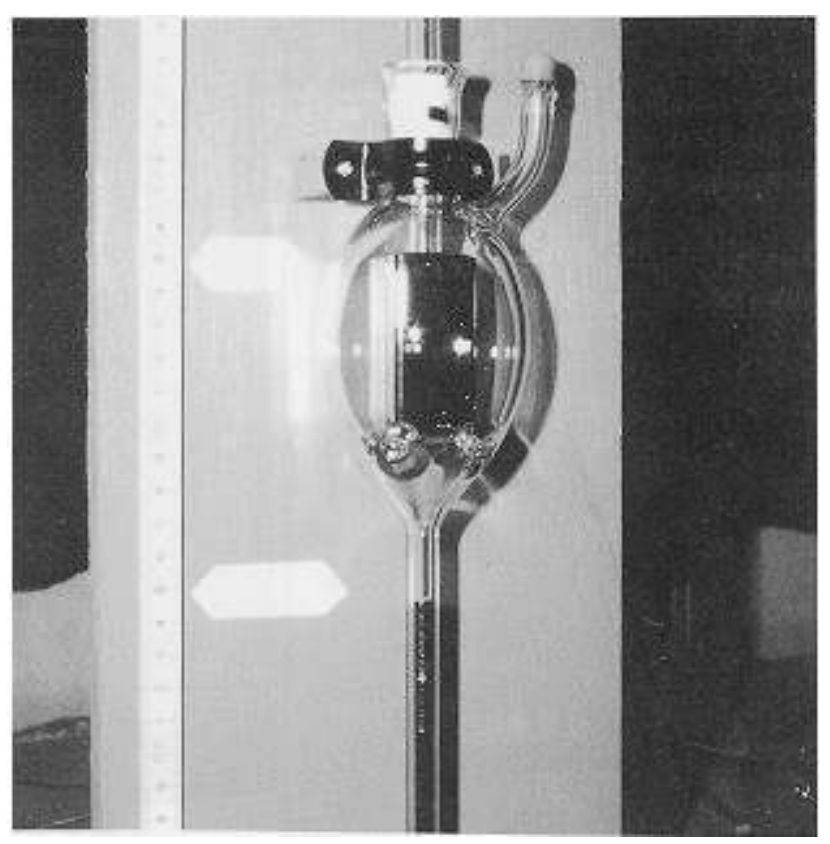

Ibid., p. $769-770$ : 
[...] si l'aide retire le doigt qu'il tient au-dessous, plongé, comme auparavant, dans le vif-argent étendu en nappe à l'extérieur en $\mathrm{D}$, tu remarqueras avec admiration un équilibre permanent du vif-argent $\mathrm{AE}$ contenu dans le tube inférieur avec l'air extérieur; le tube situé en haut se videra entièrement par les côtés du vase intérieur $\mathrm{C}$ : le vif-argent débordera et le tube $\mathrm{AE}$ qui porte le flacon en retiendra vingt sept pouces.

Introduction d'air par le trou d'une aiguille : deuxième étape

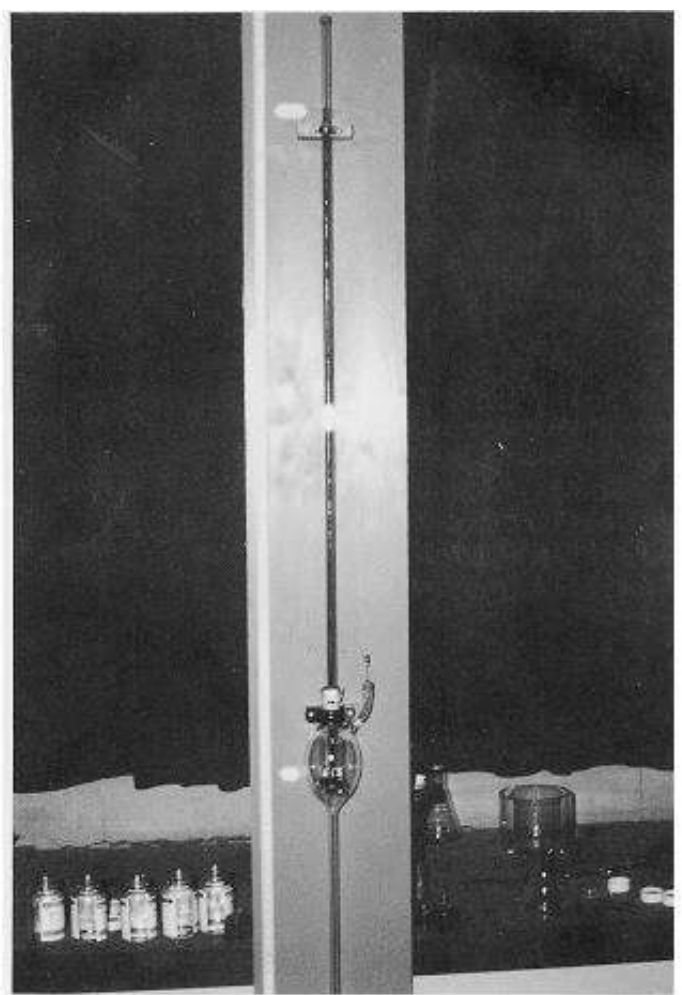

Ibid., p. 770 :

Si alors, avec une aiguille très fine, tu perces la vessie de l'orifice $G$, et laisses un peu d'air pénétrer à l'intérieur, celui-ci se mélange avec celui qui se trouve dilaté dans le flacon et en augmente la pression, de sorte que, dès lors, il exerce de tous côtés une poussée plus forte : il presse le vif-argent du bas, situé dans le col AE et le fait s'abaisser sensiblement, en même temps qu'il comprime celui qui repose dans le vase intérieur $\mathrm{C}$ et l'oblige à monter dans le tube supérieur $\mathrm{CF}$ en formant un cylindre bien apparent ; bien plus, au fur et à mesure que l'air pénètre, tu le vois croître progressivement jusqu'à la hauteur de vingt-sept pouces du côté de $F$, tandis que le vif-argent du tube inférieur s'affaisse complètement.

\section{L'expérience de Roberval}

L'expérience de Roberval est connue par la Gravitas comparata du P. Noël ; le texte est cité d'après l'édition des Euvres de Pascal par J. Mesnard, II, p. 637-639. 


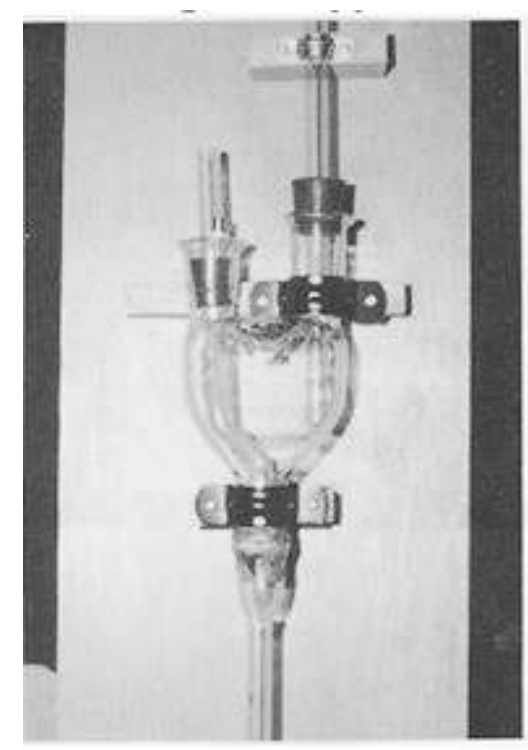

Schéma du montage de l'appareil

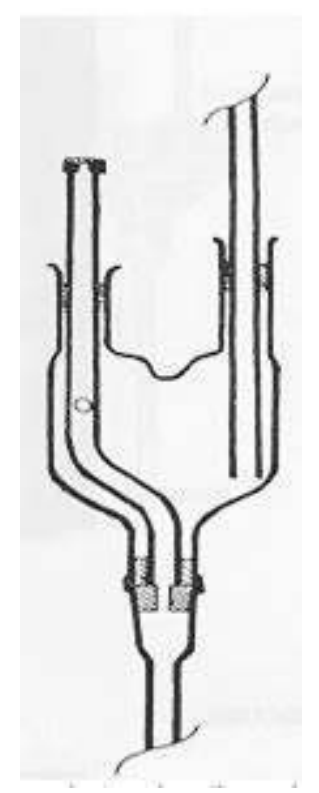

Ibid., p. 637-638:

Soit un tube de verre long de trois pieds, ouvert à chaque extrémité. Soit un flacon de verre, enforme de cœur humain, par la base duquel deux petits tubes, ouverts à chaque extrémité, l'un à droite, l'autre à gauche, pénètrent de telle manière que celui de droite, perpendiculaire, arrive, par son extrémité inférieure, au voisinage $\mathrm{du}$ fond $\mathrm{du}$ flacon, et, par son extrémité supérieure, dépasse la base de vingt-sept pouces ; que celui de gauche, qui dépasse le flacon d 'un ou deux pouces, s'écarte de la perpendiculaire, et qu'il traverse le col du flacon par le milieu de sorte qu'à son extrémité inférieure il devienne perpendiculaire et s'avance assez loin au-delà du flacon. Entre la base et le col du flacon, qu'un trou y soit ouvert au milieu. Enfin, que le flacon soit si bien fixé au tube décrit le premier que, par l'extrémité où ils communiquent, aucune autre voie d'accès ne s'ouvre que par le tube de gauche. 
Introduction du mercure, versé dans un petit tuyau de gauche recourbé

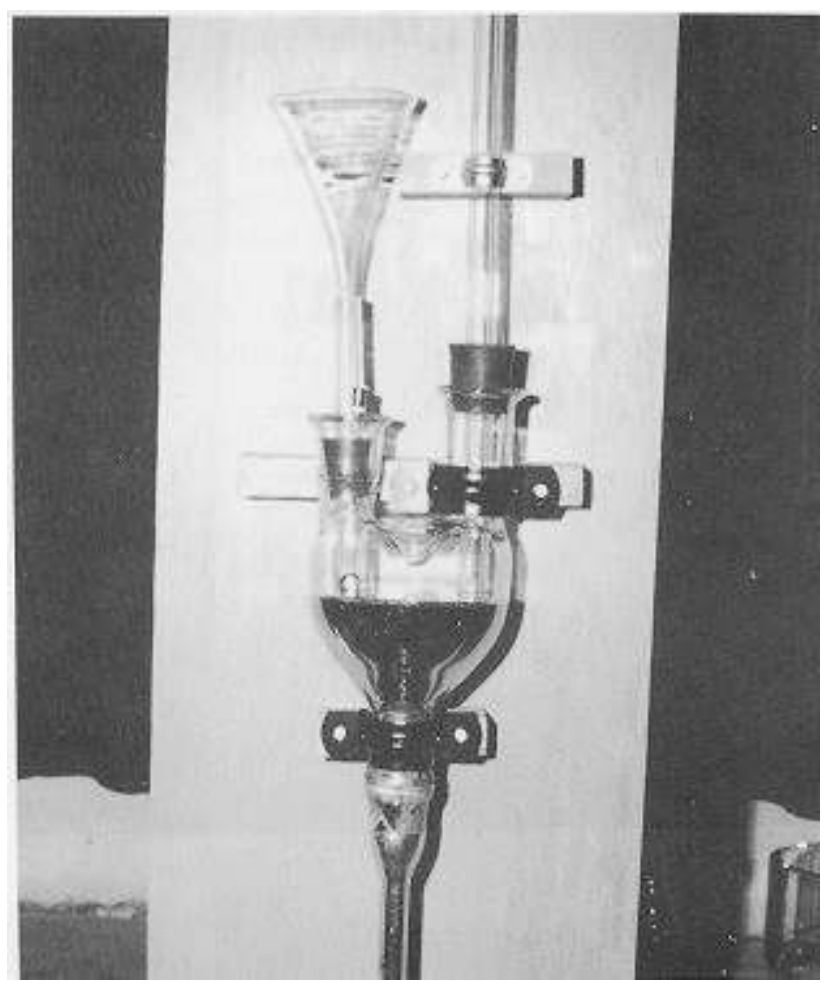

Ibid., p. 638 :

Le dispositif étant ainsi monté, d'abord, soit fermée par une membrane la seconde extrémité du long tube ; puis, par le tube recourbé, celui de gauche, soit versé du vif-argent, dont soient remplis le tube, la partie inférieure du flacon et le petit tube. Soit alors bouché le petit tube, à son extrémité supérieure, par une membrane.

Ajout de cire pour combler l'interstice entre le tuyau de gauche et le flacon 


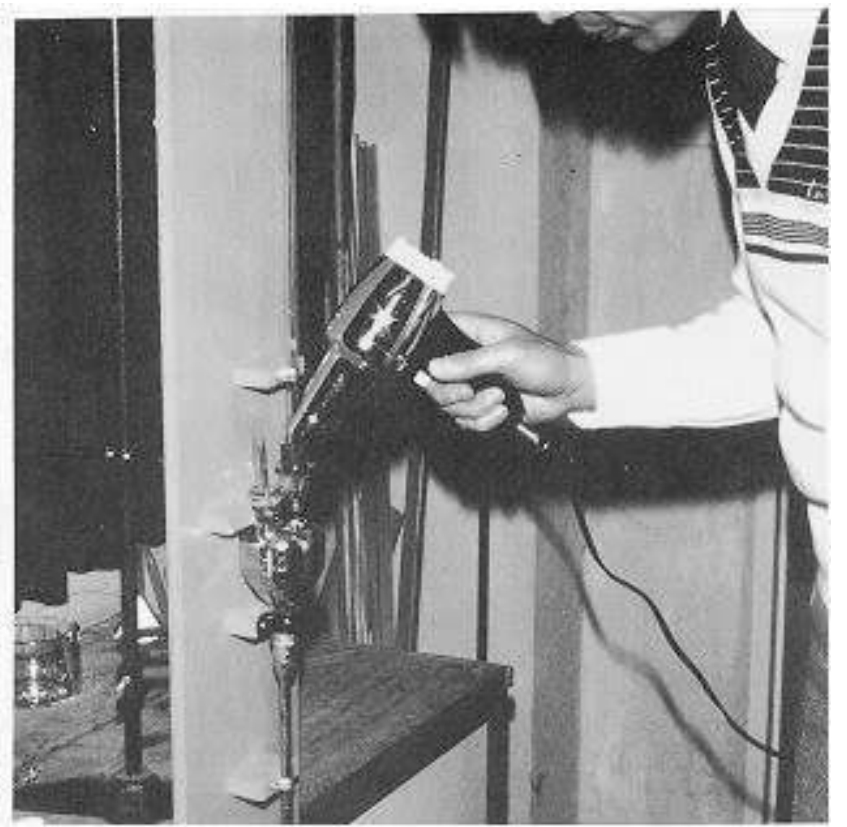

Commentaire: Malgré le texte de Noël cité plus haut: «aucune autre voie d'accès ne s'ouvre que par le tube de gauche ", il faut laisser ouverts les deux joints entre le flacon et les deux petits tuyaux, faute de quoi on ne pourra pas remplir la partie supérieure du flacon.

30 Lorsque le flacon est presque rempli, nous avons enfoncé le bouchon en gomme dans le joint de gauche et comblé l'interstice avec de la cire en la faisant fondre à la chaleur.

Flacon complètement rempli de mercure

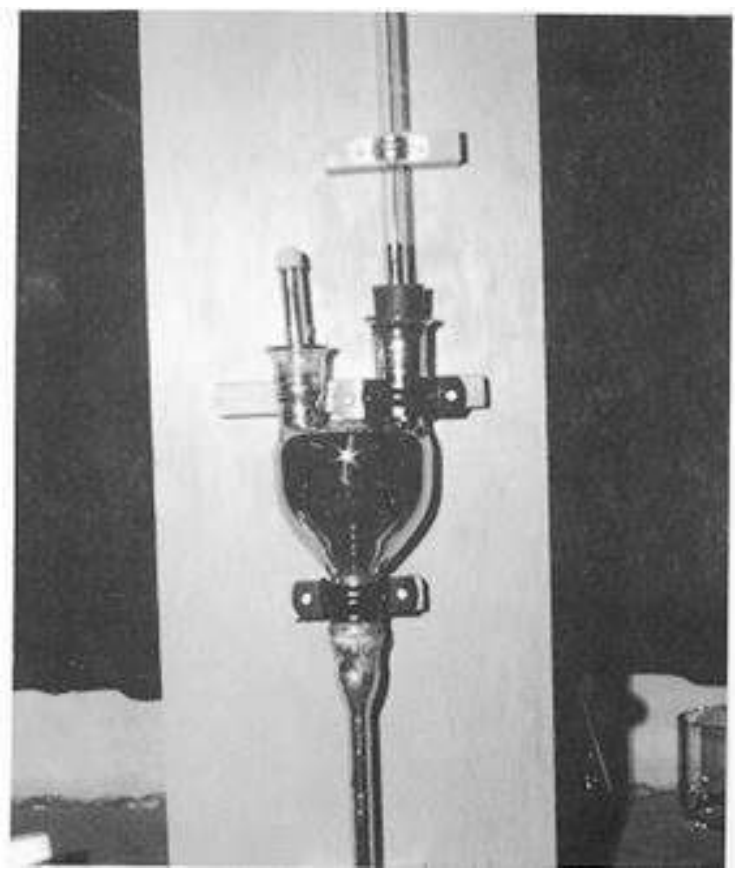


31 Commentaire: Ayant achevé de remplir le flacon, on bouche l'extrémité supérieure du petit tuyau de gauche. Puis on enfonce le bouchon dans le joint de droite et on comble l'interstice entre le tuyau de droite et le flacon avec de la cire.

Dispositif complètement rempli de mercure : étape préparatoire

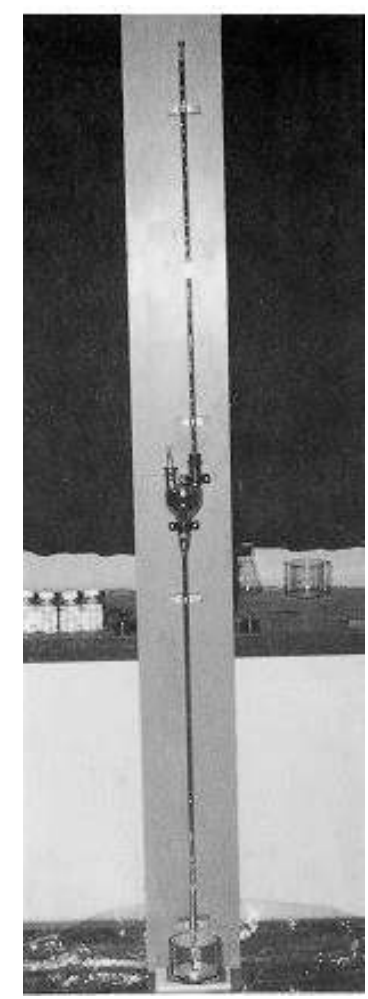

Schéma du dispositif complétement rempli de mercure

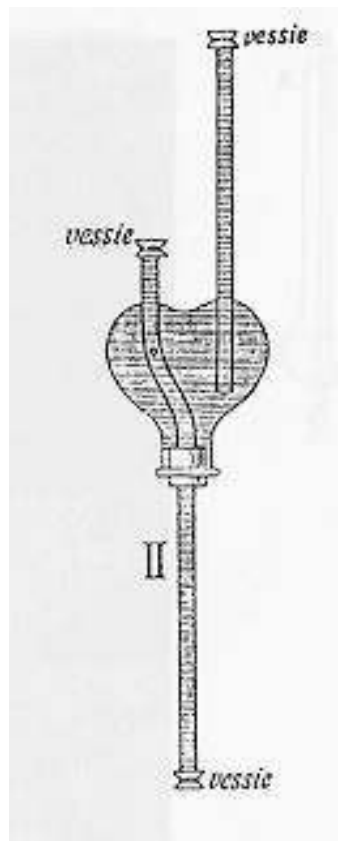

Ibid., p. 638 : 
Enfin soient le petit tube de droite, et la partie supérieure du flacon remplis de vif argent, et soit le petit tube fermé par une membrane.

Voilà les vases dont on dispose : le long tube, le flacon et les petits tubes tous pleins de vif-argent.

Soit le grand tube, du côté où il est bouché par une membrane, plongé dans le vif argent d'un récipient placé au-dessous, et soit ce tube, avec le flacon et les petits tubes, dressé perpendiculairement.

Ouverture du tube par l'extrémité inférieure : première étape

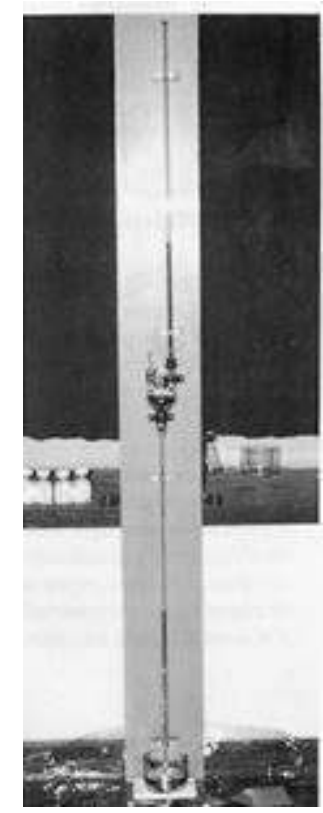

Schéma du dispositif

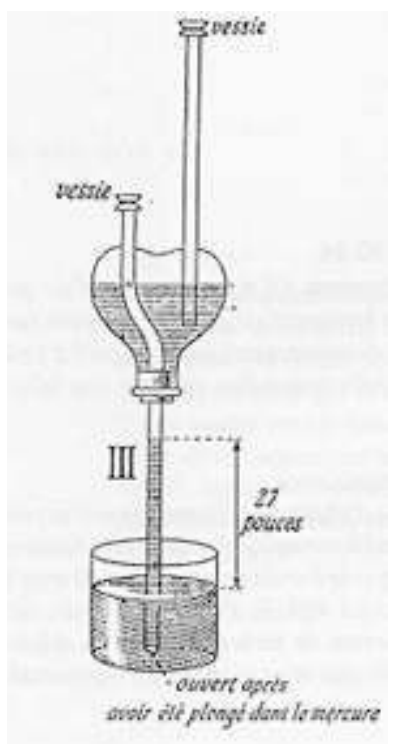

Ibid., p. 639 :

Soit débouchée l'extrémité inférieure du grand tube. Le vif-argent descend, [...] du sommet du petit tube de droite, à la place du vif-argent qui descend, jusqu'à ce que celui-ci parvienne à la hauteur de vingt sept pouces au-dessus de la surface du vif- 
argent du récipient placé au-dessous du long tube : dans la mesure où le flacon se vide de vif-argent, [...]

32 Commentaire: La confrontation de notre photo au troisième dessin de Duhem montre clairement notre échec dans cette étape. À l'étape préparatoire déjà, le dispositif n'était pas bien rempli : il restait de l'air en haut du tube. Nous avons donc recommencé le remplissage du dispositif.

Introduction d'air par le tuyau de gauche : deuxième étape

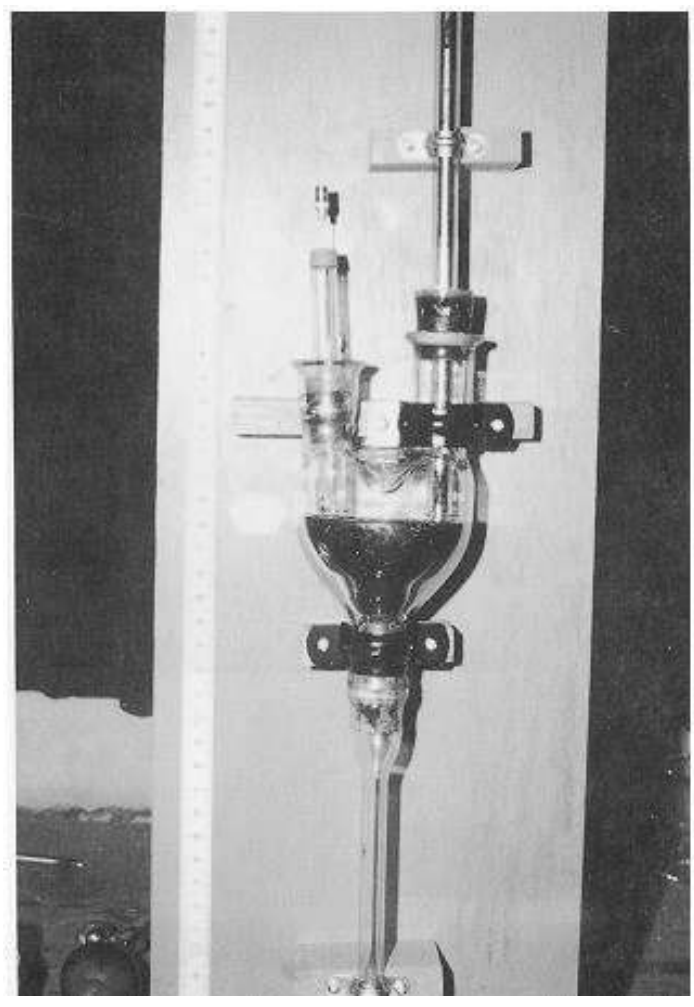


Schéma du dispositif

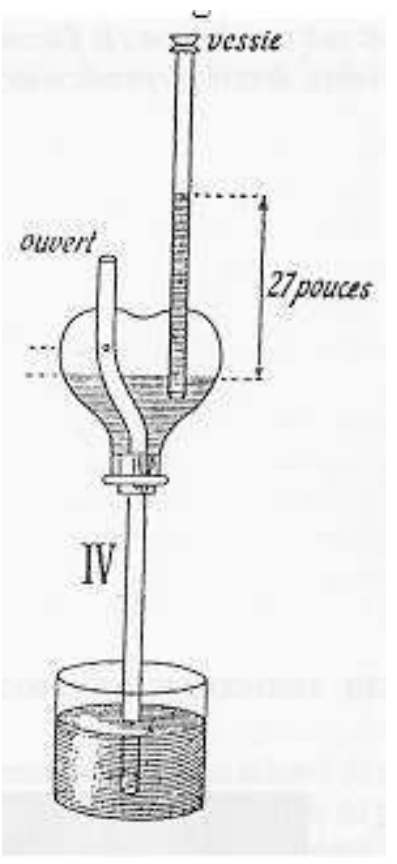

Ibid., p. 639 :

Si l'on perce la membrane du tube de gauche, celui qui est recourbé, de manière à ouvrir un étroit accès à l'air, l'éther est chassé du petit tube de droite par l'air qui se répand tout autour, le vif-argent est chassé du flacon pour prendre la place de l'éther, et l'air prend la place du vif-argent.

\section{L'expérience de Rohault}

Voir Rohault, Traité de Physique (1671), p. 92-95.

Que le vif-argent du tuyau descendrait entièrement, s'il n'y avait point d'air grossier qui pesât sur le vaisseau.

Tout ainsi donc que lorsqu'il y a une moindre hauteur d'air grossier qui pèse sur le vif-argent du vaisseau, on conclut que celui du tuyau s'y doit trouver à une moindre hauteur, de même aussi, supposé qu'il n'y eût point du tout de cet air grossier qui pesât dessus, on doit conclure que le vif-argent doit descendre tout-à-fait, en sorte que celui du tuyau soit de niveau avec celui qui est dans le vaisseau.

Description d'une machine pour faire cette expérience

Quelques-uns ont estimé qu'il était impossible d'observer si l'expérience s'accorde en cela avec le raisonnement, tant à cause qu'il n'y a point de montagne assez haute pour nous élever au-dessus de la plus haute surface de l'air, qu'à cause que quand il y en aurait, on n'y pourrait pas subsister, parce que l'on y respirerait un air qui serait trop subtil. Mais je me suis avisé d'un moyen qui a levé ces deux difficultés, et par lequel il ne m'a pas été difficile d'en venir à bout; ce moyen consiste dans la construction d'une espèce de chambre, dont les murailles sont transparentes, de sorte qu'on peut du dehors regarder sans danger ce qui se passe au dedans. J'ai donc fait faire une machine de verre, telle que vous la voyez ici représentée [...]. 
Partie centrale de l'appareil

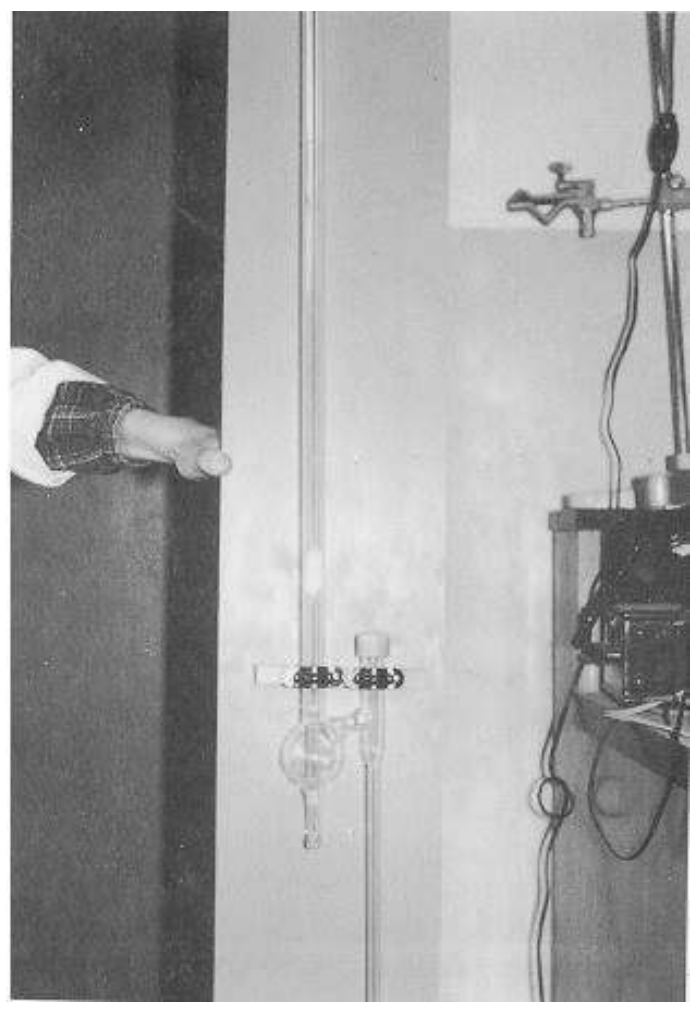

Schéma de la partie centrale de l'appareil

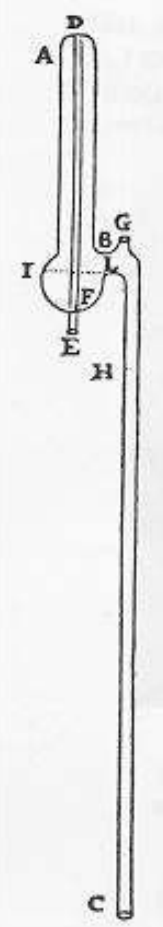

Ibid., p. 92-93 : 
$B C$ est un tuyau qui a plus de vingt-sept pouces et demi, et qui est ouvert vers $C ; A B$ est une grande cavité, qui a communication avec BC, par sa partie BL, et qui est fermée du côté de $A$; DB est un petit tuyau de verre fermé par le bout $D$, et qui sortant hors de la chambre, ou cavité $\mathrm{AB}$, par sa partie $\mathrm{FE}$, est ouvert par le bout $\mathrm{E}$; outre cela, ce petit tuyau a un petit trou vers $F$, où il est soudé extérieurement avec le verre $A B$, en sorte que la cavité du petit tuyau a communication avec la grande cavité $\mathrm{AB}$, par ce petit trou $\mathrm{F}$. Enfin, il y a encore un bout de tuyau $\mathrm{BG}$, par où l'air extérieur peut avoir communication avec celui qui est dans le tuyau $A B C$.

Renversement du dispositif et introduction du mercure dans l'appareil

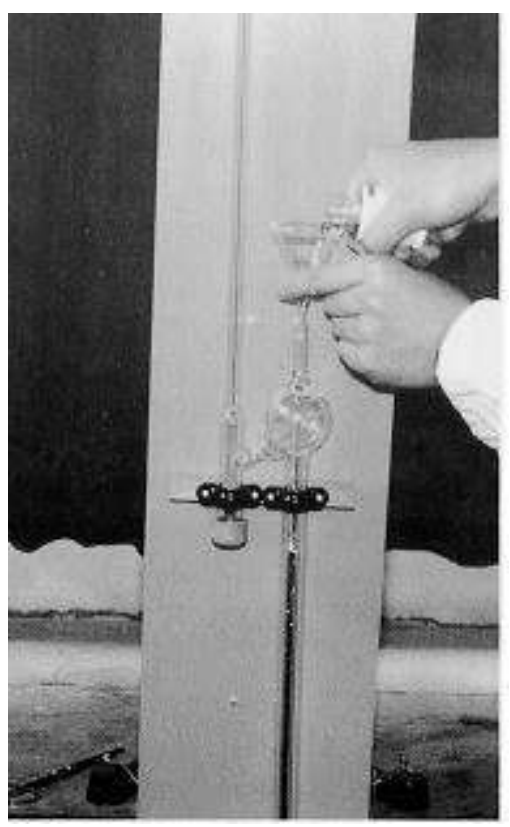


Schéma correspondant

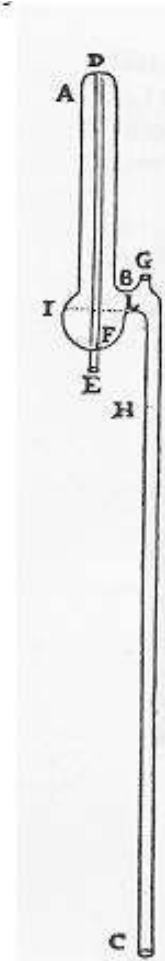

Ibid., p. 93 :

Je bouche d'abord l'ouverture $\mathrm{G}$ avec de la vessie de porc; puis renversant tout le tuyau, en sorte que le bout $C$ soit tourné vers le haut, je verse du vif-argent par le trou $\mathrm{E}$, lequel au commencement tombe seulement dans le petit tuyau DFE ; [...]

Vue du dispositif

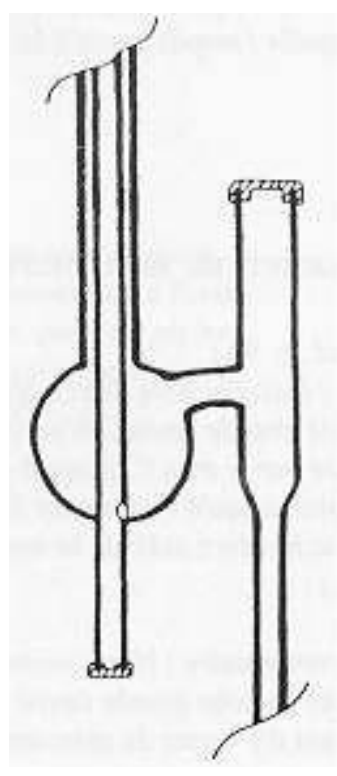


Introduction progressive du mercure dans la cavité $A B$ jusqu'à ce que cette dernière soit pleine

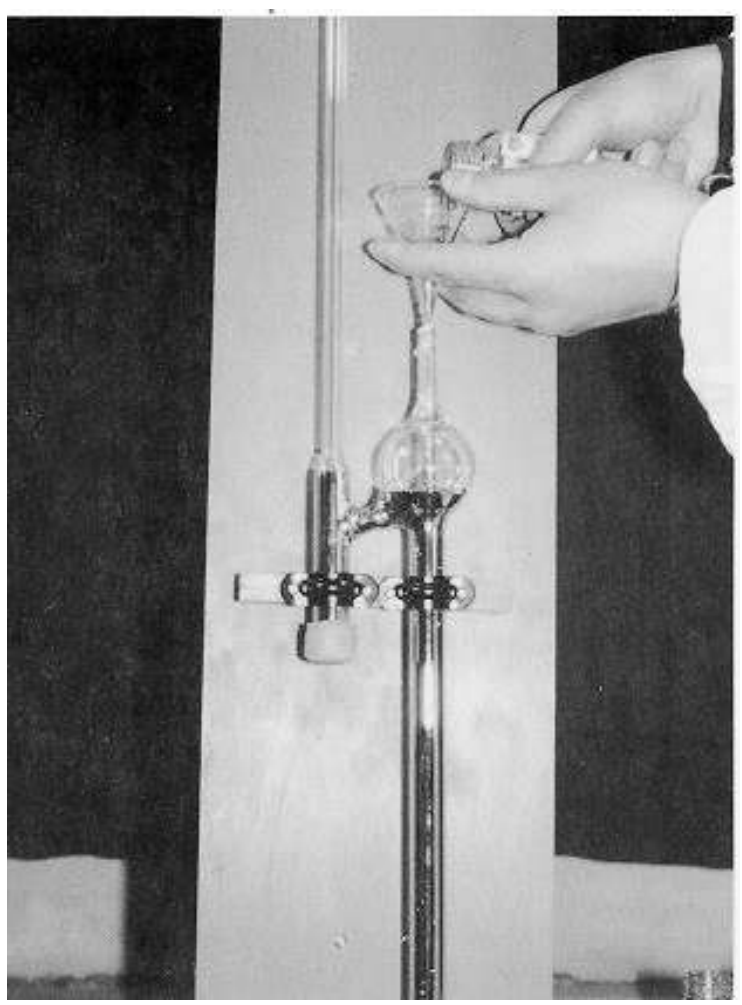

Ibid., p. 93 :

[...] mais quand il est plein jusqu'à $F$, celui que je continue de verser tombe par ce trou qui est en cet endroit-là, et va remplir la cavité $\mathrm{AB}$, qui est autour de ce petit tuyau, laquelle j'emplis jusqu'à la hauteur B ; [...]

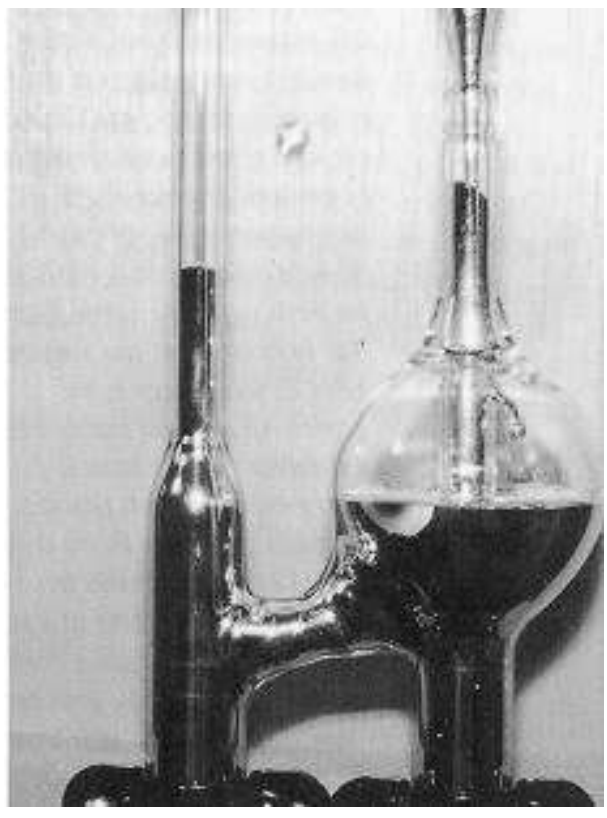




\section{Fermeture de l'orifice $\mathrm{E}$}

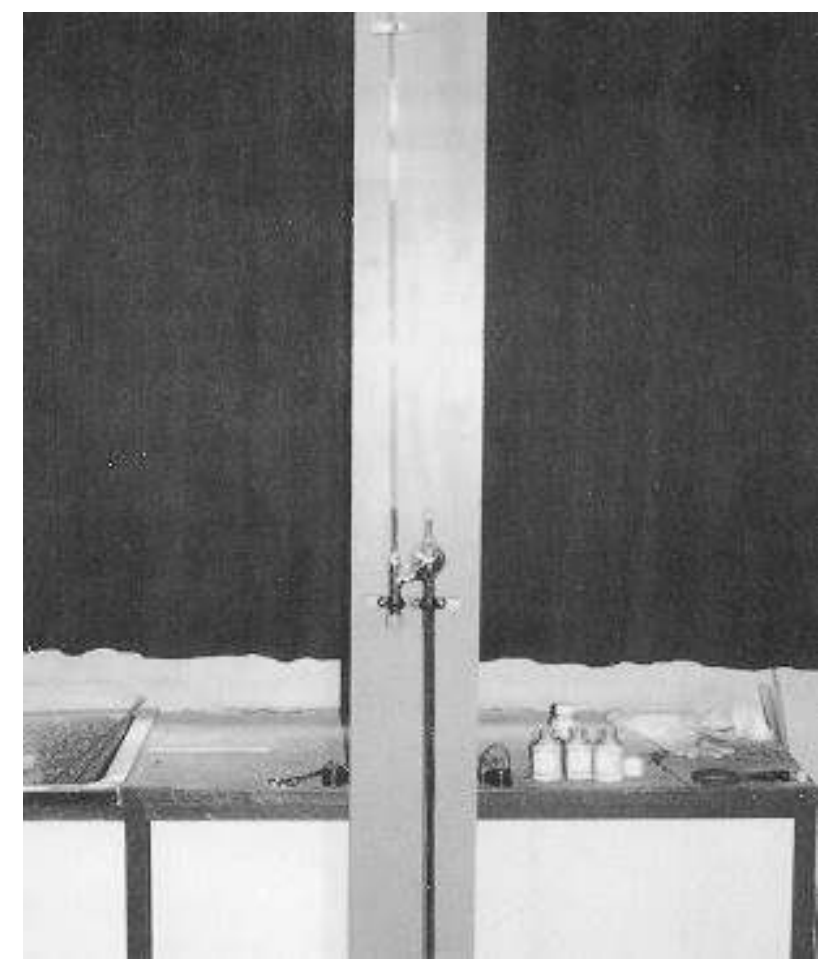

Ibid., p. 93 :

[...] Puis j'achève de remplir le reste de cette grande cavité, en versant du mercure par le trou C, jusqu'à ce qu'il soit monté jusqu'à l'ouverture $\mathrm{E}$, que je bouche alors avec de la vessie de porc; $[$...]

Commentaire: Nous avons rempli «le reste de cette grande cavité » en continuant d'y verser du mercure par l'orifice $\mathrm{E}$.

Quant au remplissage de l'appareil, il est possible de l'effectuer dans différents ordres. On peut, par exemple, remplir tout l'appareil en ne se servant que de l'orifice C, sauf la partie EF.

41 Nous avons introduit du mercure par l'orifice E le plus longtemps possible afin de protéger la capsule de l'ouverture $\mathrm{G}$ contre le choc du mercure qui tombe du haut du tuyau CG. Il nous semblait aussi plus facile de pouvoir verser du mercure sans avoir à monter sur l'escabeau. 
Introduction du mercure par l'orifice C

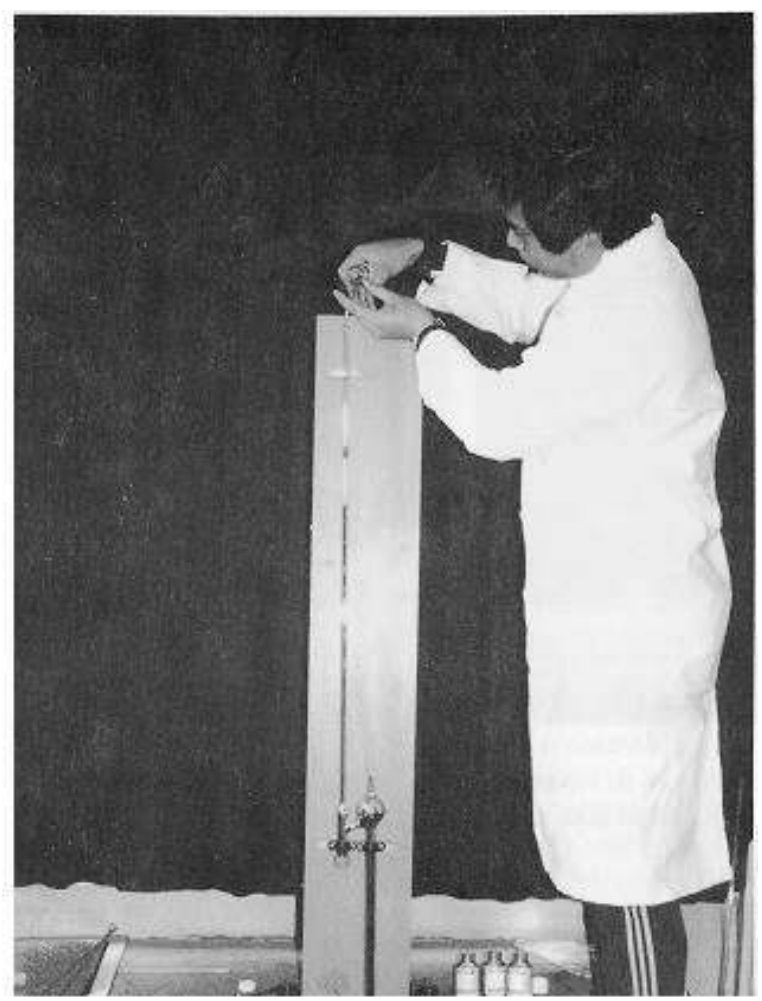

42 Ibid., p. 93 :

[...] après quoi, continuant de verser du vif-argent par le trou $C$, j'achève de remplir entièrement le tuyau $\mathrm{BC}$. [...] 
Dispositif complètement rempli : étape préparatoire

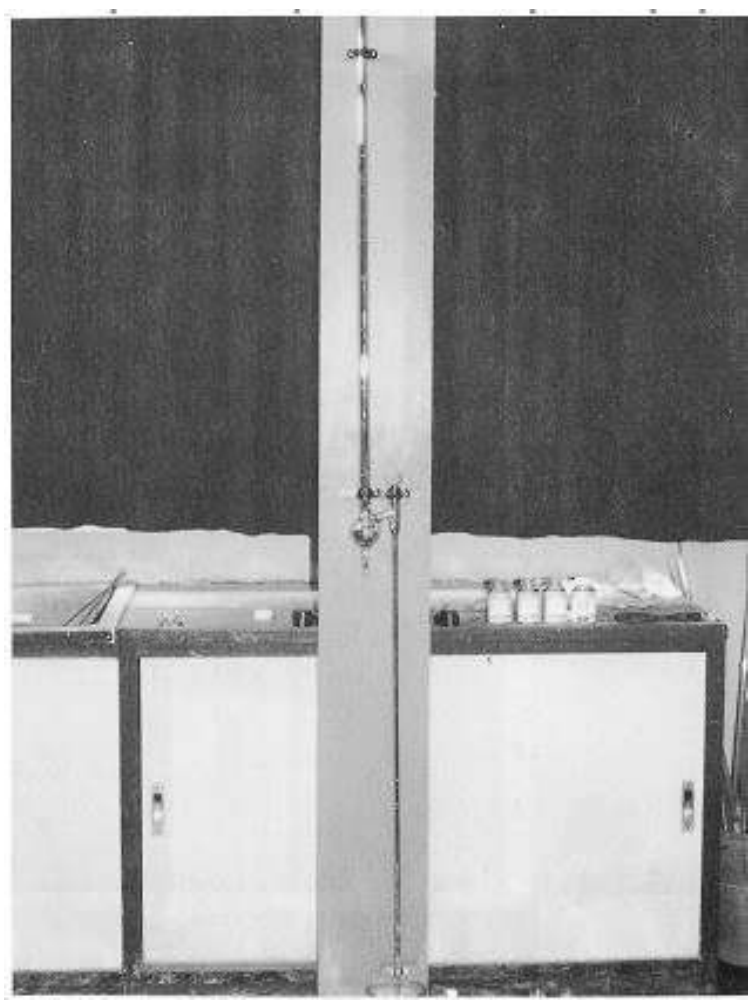

$43 \quad$ Ibid., p. 93-94 :

[...] Cela fait, je bouche avec le doigt cette ouverture $\mathrm{C}$, et renversant à l'ordinaire toute la machine, qui n'est pleine que de vif-argent. je la plonge dans un vaisseau où il y en a déjà ; [...] 
Ouverture du tube par l'extrémité inférieure

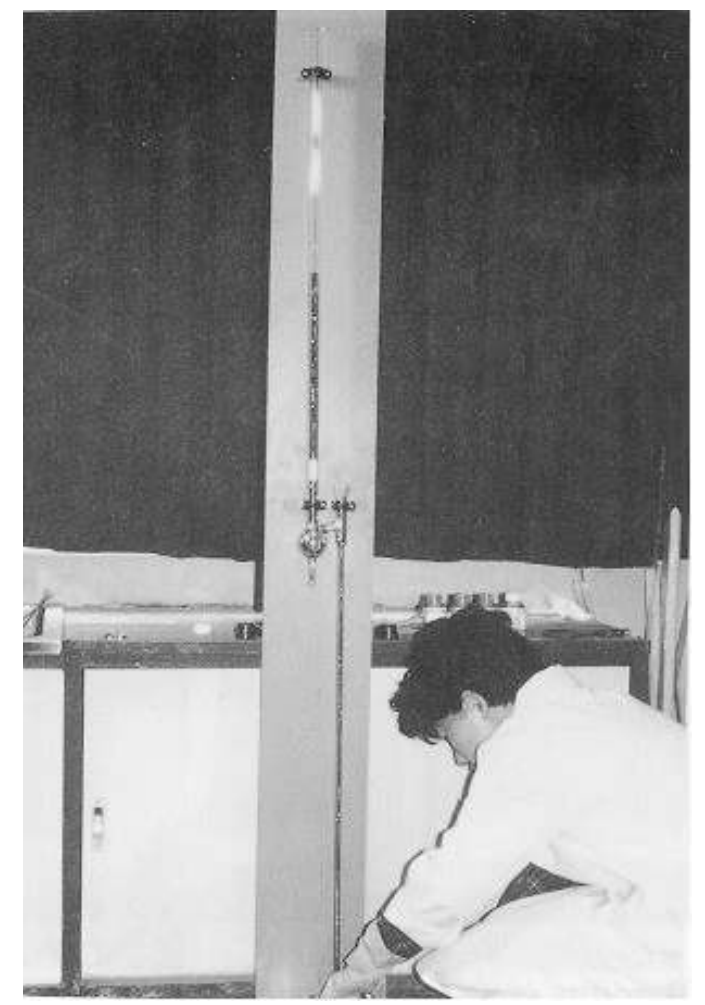

\section{Première étape}

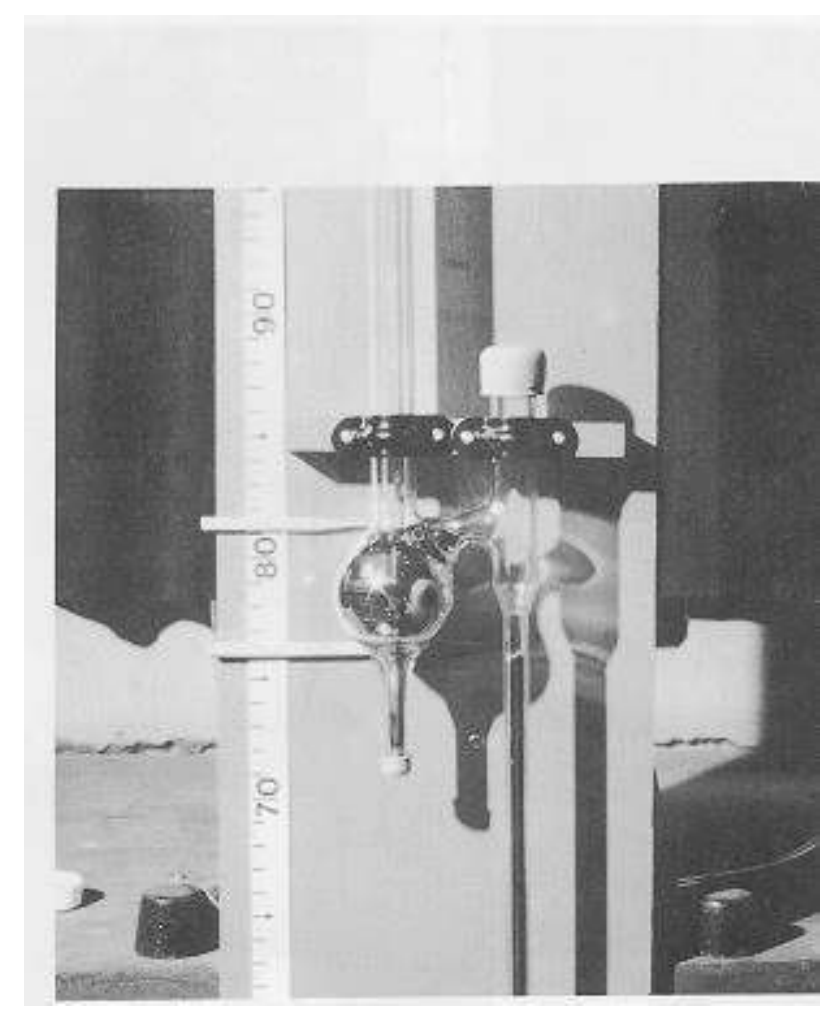

44 Ibid., p. 94 : 
[...] Et alors la capacité AF se vide jusqu'à IL, et en même temps le petit tuyau DFE se vide aussi jusqu'à pareille hauteur, et le tuyau $C$ se vide jusqu'à $H$, qui est élevé pardessus le vif-argent du vaisseau de vingt-sept pouces et demi. Ainsi l'on voit la conformité de l'expérience avec le raisonnement : car comme aucun air grossier ne pèse sur la surface IL du vif-argent qui s'est réservé dans le creux IFL, aussi rien ne le force à monter dans le petit tuyau DFE. [...]

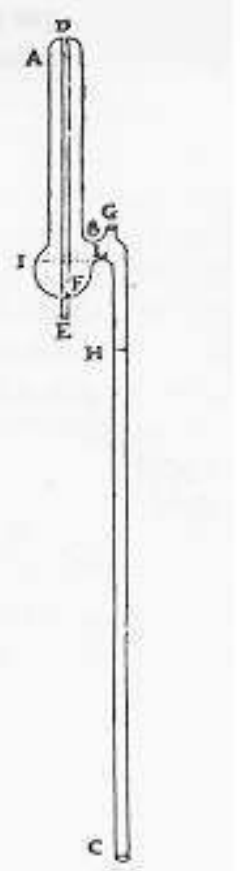

Introduction d'air par l'orifice G

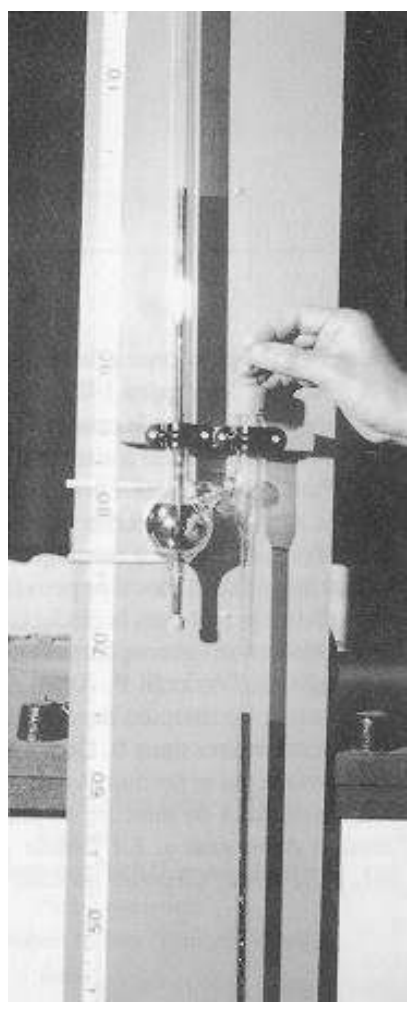


Ibid., p. 94 :

[...] Ensuite de ceci, si l'on perce avec une épingle la vessie de porc qui bouche l'ouverture G, il est évident que l'air grossier qui entrera dans la capacité $A B G$ doit produire deux effets tout différents, et pour cela même fort remarquables; le premier est que pesant sur le vif-argent qui est directement au dessous de $G$, il le forcera de descendre ; et d'ailleurs pesant aussi sur la surface IL du vif-argent qui s'était réservé dans le creux IFL, il en contraindra une partie de monter dans le petit tuyau DFE; $[\ldots]$

\section{Deuxième étape}

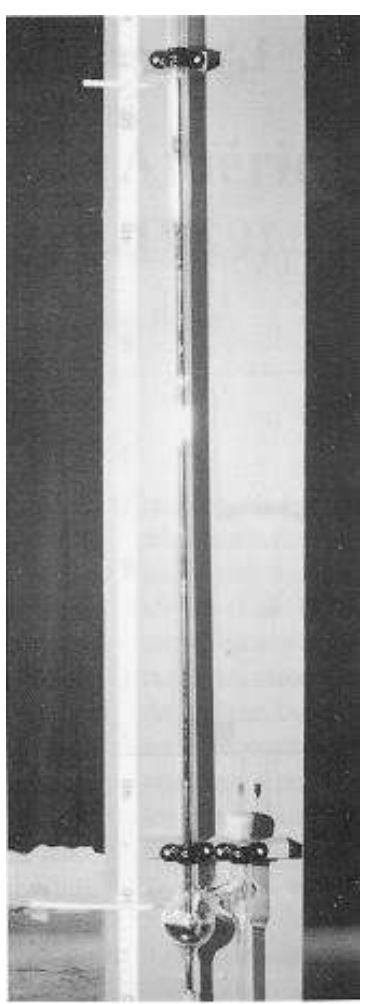

Ibid., p. 94 :

[...] et même il le remplira tout-à-fait, pourvu que sa hauteur n'excède pas vingtsept pouces et demi. [...] 


\section{Étape supplémentaire}

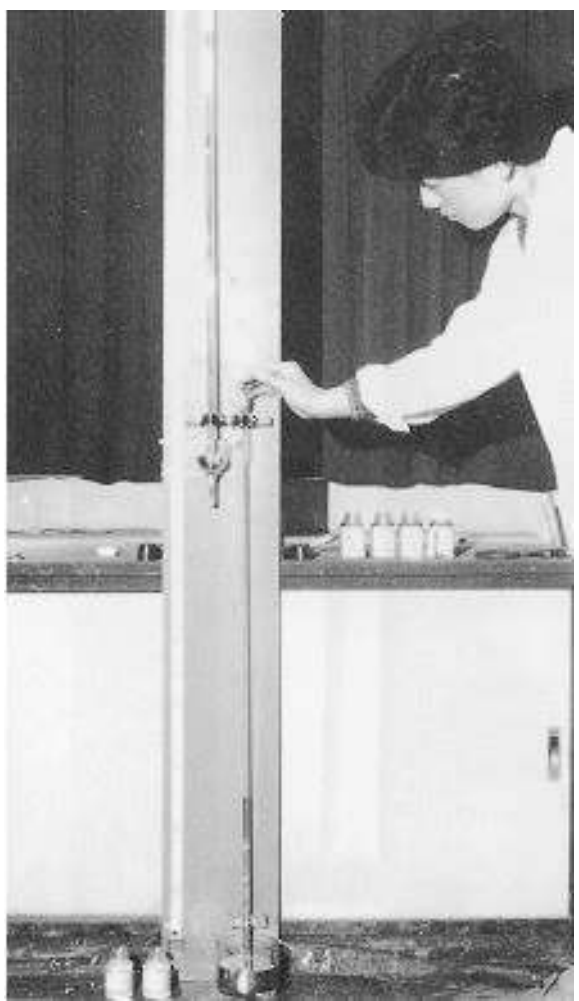

\section{Ibid., p. 94-95}

[...] Pour avoir plus de plaisir en faisant cette expérience, après avoir percé avec une épingle la vessie de porc. qui bouche l'ouverture $G$, il faut à diverses fois retirer tant soit peu l'épingle pour laisser entrer à chaque fois un peu d'air par l'ouverture, et aussitôt le renfoncer, et alors vous aurez le plaisir de voir monter peu à peu, et à diverses reprises le vif-argent dans le petit tuyau DFE, tandis qu'il descend aussi peu à peu dans le tuyau $\mathrm{DFE}$, tandis qu'il descend peu à peu dans le tuyau $\mathrm{BC}$. Puis il faut tout d'un coup enlever tout à fait l'épingle, et alors vous le voyez en même temps monter d'un côté, tandis qu'il descend de l'autre.

\section{BIBLIOGRAPHIE}

Koyanagi Kimiyo, « Pascal et l'expérience du vide dans le vide ", Japanese Studies in the History of Science, $\mathrm{n}^{\circ} 17,1978$.

Uchida Masao, « De la faisabilité des « Expériences du vide dans le vide » rapportées par Pascal et ses contemporains ", Bulletin de la Faculté des Lettres et Sciences Humaines de l'Université Wako, $\mathrm{n}^{\circ} 17$, 1982. 
INDEX

Mots-clés : physique, vide

Keywords : physics, vacuum 